Source: Barkhordari, S. Alizadeh, A., Yadollahi, M. \& Namazi, H. (2020). One-pot synthesis of magnetic chitosan/iron oxide bionanocomposite hydrogel beads as drug delivery systems. Soft Materials. https://doi.org/10.1080/1539445X.2020.1829642.10.1080/1539445X.2020.1829642.

\title{
One-pot synthesis of magnetic chitosan/iron oxide bionanocomposite hydrogel beads as drug delivery systems
}

\author{
S. Barkhordari ${ }^{\mathrm{a}}, \underline{\text { A. Alizadeh }}^{\mathrm{a}, \mathrm{b}}$, M. Yadollahi ${ }^{\mathrm{c}} \& \mathrm{H}$. Namazi $^{\mathrm{c}}$
}

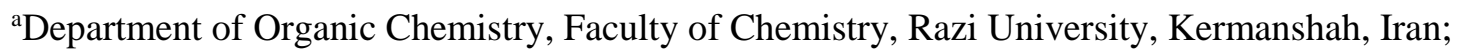

bNanoscience \& Nanotechnology Research Center (NNRC), Razi University, Kermanshah, Iran;

${ }^{c}$ Laboratory of Dendrimers and Biopolymers, Faculty of Chemistry, University of Tabriz, Tabriz, Iran
\end{abstract}

\begin{abstract}
In this research, a novel dual-responsive supermagnetic chitosan/iron oxide nanocomposite hydrogel beads $(\mathrm{CH} / \mathrm{MION})$ was prepared using sodium tripolyphosphate (STPP) as cross linking agent. The prepared CH/MION hydrogel was systematically characterized by Fourier transforminfrared spectroscopy (FT-IR), scanning electron microscopy (SEM) X-ray diffraction (XRD), and vibrating sample magnetometer (VSM). In addition, the $\mathrm{CH} / \mathrm{MION}$ hydrogel exhibits favorable swelling properties that are sensitive to $\mathrm{pH}$, and external magnetic field (EMF). The drug release behavior of the prepared hydrogel under EMF, and different $\mathrm{pHs}$ was also studied. The obtained hydrogel showed considerable temperature, $\mathrm{pH}$, and EMF sensitivity for the in vitro release of Doxorubicin (DOX). These results suggested that the $\mathrm{CH} / \mathrm{MION}$ hydrogel beads could be a promising candidate for drug deliver applications.
\end{abstract}

Keywords: drug delivery, chitosan bead, iron oxide nanoparticle, bionanocomposite 